\title{
NUMERICAL STUDY AND DYNAMIC BEHAVIOR ANALYSIS OF MECHANICAL HEART VALVE
}

\author{
CHEUNG-Hwa HsU \\ Department of Mechanical Engineering, \\ Southern Taiwan University of Technology, Tainan, Taiwan
}

\begin{abstract}
This paper develops an analysis tool, for the mechanical heart valve designs, which contains a quick flow analysis model and a pseudo steady-state dynamic model. The flow model utilizes an irrotational, inviscid algorithm of source/doublet elements simulating the closed leaflet geometry and the aortic root coupled with a boundary layer model to simulate the viscous components. The numerical study provides quick simulation and vital design data of the shear stress distributed over the valve leaflet, the separation point prediction behind the leaflet and the sewing ring, and the aerodynamic loads acting on the leaflet. Those aerodynamic loads have been sent to a dynamic model, which simulates the leaflet motion as a $2^{\text {nd }}$ order rotating system, to simulate the opening/closing characteristics of the leaflet. The analysis of the presented new heart valve design leads to a conclusion that the regurgitant volumes depends very much on the orientation of the gravity force. The maximum difference of the ratio of the valve closing regurgitation to the main flow can be as high as $12 \%$. Hence the leaflet ought to be designed with a light material to avoid the unstable influence on the heart work due to the human's physical actions.
\end{abstract}

Biomed Eng Appl Basis Comm, 2001 (December); 13: 283-295.

Keywords: mechanical heart valve, flow analysis, dynamic model, regurgitant volume

\section{INTRODUCTION}

The first prosthetic heart valve to be implanted clinically was a mitral valve with a caged ball design in 1960 [1], and subsequently also for aortic valve replacement. Till now, many varieties have come into commercial use. At present, there are two basic categories of artificial heart valves, one is a mechanical heart valve and the other is a tissue valve. The tissue valve is manufactured from biological tissue, it incorporates most of the characteristics of an ideal heart valve substitute, e.g. a central flow orifice, low thrombogenic potential in the absence of anti-coagulation

Received: Oct. 21, 2001; accepted: Nov. 8, 2001 Correspondance: Cheung-Hwa Hsu

Department of Mechanical Engineering, Southern Taiwan University of Technology, Tainan, Taiwan therapy. However, this valve has the drawback of poor long-term durability. The mechanical heart valve, on the other hand, is manufactured with rigid leaflets from bio-inert material, e.g. pyrolytic carbon. This valve has good durability but poor haemodynamic characteristics. Five clinical conditions have been identified as being potential causes of failure of mechanical heart valves [21], they are haemolysis due to high in-bulk shear stress, shear stress related haemolysis at the blood/prosthesis interface, damage to endothelial linings due to jet impingement, valve dysfunction due to thrombus formation at pivot points, thrombo-embolism due to local stasis flow, possibly, downstream of the valve leaflets or sewing ring. Due to these clinical problems, a constant anti-coagulation treatment is required for the recipients of mechanical heart valves. There is therefore a requirement to design a mechanical heart valve which has good long-term durability but with a low anti-coagulation therapy requirement. This would need a detailed analysis for heart flow.

There are a number of in vitro techniques, e.g. laser Doppler anemometry, ultra-sound Doppler, flow 
visualization, used for studying heart flow and extracting information from the flow. Each has its own special difficulties and advantages and each can give only certain data. It is therefore necessary to use several techniques in a complementary manner to gain more aspects of flow behavior around heart valves. On the other hand, the numerical simulation of flow around prosthetic heart valves were being generated as early as 1970's [9]. In the past decades, the computational fluid dynamics (CFD) were broadly utilized for the computational analysis of prosthetic heart valves for a range of Reynolds numbers using finite clcment and finite difference methods [7]. It is noted that CFD can yield full field information about any flow parameters including those yielded by in vitro techniques and those cannot. With the improvement in computer hardware and sofiware, CFD has become a more powerful tool not only for heart valve analysis but also for heart valve design. McQueen and Peskin [15] have developed a computer-assisted design model for mitral valve design. They suggested that modest curature of leaflets can make a substantial improvement in the performance of mitral valves. More recently, they have generated a three dimensional computational method for blood flow in the heart which includes the heart contractilc fibers [16]. These studics illustrate the usefulness of computer testing in analyzing the mechanical propertics of mechanical heart valve during the design cycle.

For the dynamic behavior analysis of mechanical heart valves, very few studics have been reported. The opening/closing characteristics of the valve leaflet mainly depend upon the dynamic interactions between the leaflets and the fluid. Prabhu and Hwang [18] considered the fluttering motion of the leaflet by using the concept of lift force, and indicated that the fluttering motion of the leaflet might be the result of vortex shedding at the trailing edge of the leaflet. Reif et al [19] demonstrated several mechanisms to explain the dynamic characteristics, e.g. the periodic vortex shedding, the vibration of leaflets and the dynamics of the leaflet modeled as a second order rotational system. Cheon and Chandran [4] considered monoleaflet valves such as Bjork-Shiley valve, they implemented and further developed the model of Reif et al [19] by including a model of impact dynamics between the leaflet and the guiding struts. In all of these previous works the fluid flow model had been based on simple flat plate acrodynamic theory, requiring that the leaflet be placed in an infinite medium.

On the other hand, van Steenhoven et al [20] developed an in vitro experimental study for BjorkShiley, St Jude and Hancock heart valve prostheses to concentrate on the valve closing behavior. One of their main conclusions from their experiments is that the natural valve closure is gradual, its closure already having started during systolic deceleration, while the mechanical prostheses appear to be closed mainly by the effect of the regurgitation flow in the valve during the early phase of diastole.

It should be noted that the opening/closing characteristics of the valve leaflet depend upon the dynamic interaction between the leaflets and the fluid. The interaction is highly affected by the geometry of the leaflet along with the thickness of the leaflet. As a fluid particle flows toward the leading edge of a leaflet, the pressure in the fluid particle rises from the free stream pressure to the stagnation pressure. The high fluid pressure near the leading edge impels the developing boundary layer about both sides of the bluff body (the leaflet). However, the pressure forces are not sufficient to force the boundary layers around the back side of a bluff body at high Reynolds numbers. Since the pressure increases in the direction of flow, the fluid element experiences a net pressure force opposite to its direction of motion. At some point around the bluff body the momentum of the fluid in the boundary layer is insufficient to carry the element further into the region of increasing pressure. The fluid layers adjacent to the solid surface will be brought to rest and the flow will separate from the surface. The boundary layer separation takes place near the widest section of the body from each side of the body surface and forms two free shear layers that trail aft in the flow. These two free shear layers bound the wake. Since the innermost portion of the free shear layers moves much more slowly than the outermost portion of the layers that are in contact with the free stream, the free shear layers tend to roll up into discrete, swirling, vortices. A regular pattern of vortices is formed in the wake that interacts with the body motion and is the source of the effects called vortex-induced vibration. As the flow velocity is increased or decreased so that the vortex shedding frequency approaches the natural frequency of the structure, the vortex shedding suddenly locks into the structure frequency. The lock-in resonant oscillation of the near wake, inputs energy to the structure so that large amplitude vibrations can be produced.

To analyze the dynamic interaction between the leaflets and the fluid, an analysis method of an internal flow model along with a dynamic model is established. The internal flow approach and flow separation assessment behind the leaflet is accomplished by a fluid flow model of using an irrotational, inviscid algorithm coupled with a boundary layer model. The model provides a full integration with the geometry of the heart valve and a 'quick' analysis for the flow regime of a mechanical heart valve implanted in the aorta. The flow model evaluates aerodynamic loads acting on the leaflet which is utilized in the dynamic analysis model to simulate the opening/closing behavior of the leaflet.

\section{MATERIAL AND METHODS}


Based on the data of blood samples for humans [6], the normal range of specific gravity for blood is $1.052-1.061$, the dynamic viscosity is about four times more than the dynamic viscosity of water and the peak volumetric flow rate in the heart is about $250 \mathrm{cc} / \mathrm{sec}$. The range of the Reynolds number for blood flow through blood vessel has been estimated to be $0-1500$ by using the data of the radius of the aorta [6]. Ito[11] gives the range of transition zone Reynolds number for fully developed turbulent flow in smooth wall straight pipes of circular cross section of 1500 to 2000 . Hence the majority of flow phenomenon falls into the category of the high Reynolds number laminar flow.

For high Reynolds numbers, the viscous effects are confined to thin boundary layers normally adjacent to solid bodies. Only in the thin region next to a solid boundary (the boundary layer) is the effect of viscosity important. In the region outside of the boundary layer, the effect of viscosity is negligible and the fluid may be treated as inviscid. The flow model presented in this work simulates an internal inviscid and incompressible flow modeling the human aorta such that viscous effects are constrained to lie in an infinite layer along solid or free surfaces. This type of mathematical model is numerically solved utilized the well known panel method [12]. A coupled boundary layer model is also used in simulating the flow close to the leaflet surfaces where viscous forces exerted on the blood become important. The results emanating from the coupled flow model can be directly related to important design criteria such as flow separation and dynamic response.

\subsection{Internal Flow Analysis Model}

Considering potential fluid flowing over a closed body in Figure 1, the boundaries of the flow regime can be simulated by the surface $S_{b}$ of the closed body and the wake geometry $S_{w}$. In the case of the source/doublet algorithm, surface $S_{b}$ is paneled by source/doublet elements due to the lifting nature and thickness of the body while the wake flow is modeled by only doublet elements duc to its lifting nature. By distributing the singularity elements on the surface, and placing the point $(x, y, z)$ inside the surface $S_{b}$ (Figure 1), the inner potential $\phi_{\text {in }}^{*}$ is obtained as

$\phi_{i n}^{*}(x, y, z)=\left(\phi+\phi_{\infty}\right)_{\text {in }}=\frac{1}{4 \pi} \int_{S_{s}+s_{s}} \mu \frac{\partial}{\partial n}\left(\frac{1}{r}\right) d S-\frac{1}{4 \pi} \int_{S_{s}} \sigma\left(\frac{1}{r}\right) d S+\phi_{\infty}$

here $\mu$ is the doublet strength, $\sigma$ is the source strength, $r$ is the distance between the point $(x, y, z)$ and the singularity element, $\phi$ is perturbation potential and $\phi_{\infty}$ is free stream potential. The zero flow normal to the surface boundary condition $\nabla\left(\phi+\phi_{\infty}\right) \cdot \underline{n}=0$, in terms of the velocity potential, becomes

i.e.

$$
\phi_{i n}^{*}=\left(\phi+\phi_{\infty}\right)_{i n}=\text { const } \text {. }
$$

$$
\sum_{k=1}^{N} C_{k} \mu_{k}+\sum_{-1}^{N_{k}} C_{l} \mu_{l}+\sum_{k=1}^{N} B_{k} \sigma_{K}+\phi_{\infty}=\text { const. }
$$

where

$C_{i} \equiv \frac{1}{4 \pi} \int \frac{\partial}{\partial n}\left(\frac{1}{r}\right) d S$ is the influence due to the doublet strength of panel $j$, $B_{j}=\frac{-1}{4 \pi} \int_{j}\left(\frac{1}{r}\right) d S$ is that due to the source strength,

$N_{w}$ is the number of doublet elements simulating the downstream wake, and

$N$ is the number of source/doublet elements simulating the body surface $S_{b}$.

Note that the collocation points must be placed inside the body in order to evaluate the internal velocity potential. Katz and Plotkin [12] have suggested that an inward displacement of 0.05 panel length will be sufficient.

Setting $\phi_{i n}^{*}=\phi_{\infty}$ we have that the governing equation on the collocation point becomes

$$
\sum_{k=1}^{N} C_{k} \mu_{k}+\sum_{k=1}^{N_{k}} C_{l} \mu_{l}+\sum_{k=1}^{N} B_{k} \sigma_{k}=0
$$

To define this problem uniquely, the wake doublet distribution should be known or related to the unknown doublets on $S_{b}$ and the source strength is thus required. Applying the Kutta condition

$$
\gamma_{\text {t.e. }}=\left.\frac{\partial \mu}{\partial s}\right|_{\text {t.e. }}=0
$$

along the trailing edge (refer to Figure 1); we have that $\mu$ in the wake $\left(\mu_{w}\right)$ equals the value at the trailing edge $\left(\mu_{\text {t.e. }}\right)$, i.e. $\mu_{\text {t.e. }}=\mu_{\mathrm{w}}=$ constant or $\mu_{w}-\mu_{u}+\mu_{t}=0$. Hence the wake doublet strength can be evaluated as

$$
\mu_{w}=\mu_{u}+\mu_{l}
$$

where $\mu_{\mathrm{u}}$ and $\mu_{1}$ are the corresponding upper and lower surface doublet strength at the trailing edge. The

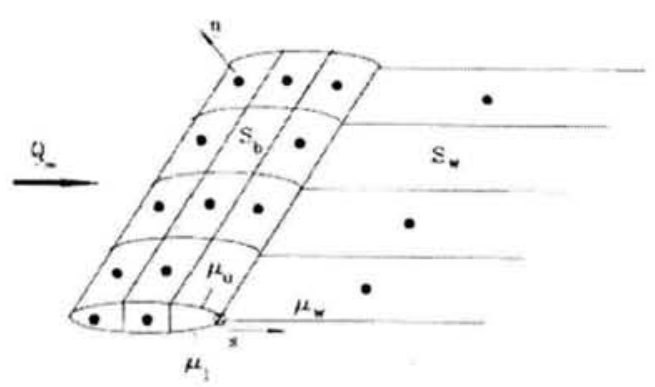

Fig. 1 A source/doublet analysis model of fluid flowing over a closed body 
value for the surface discontinuity in the normal derivative of the velocity potential is given by

$$
-\sigma=\frac{\partial \phi^{*}}{\partial n}-\frac{\partial \phi_{i n}^{*}}{\partial n}=\frac{\partial \phi}{\partial n}-\frac{\partial \phi_{i n}}{\partial n}
$$

and since $\phi_{i n}=0$ then also $\frac{\partial \phi_{i n}}{\partial n}=0$ on surface

$S_{b}$. Hence the source strength is required to be

$$
\sigma=-\underline{n} \bullet \underline{Q}_{\infty}
$$

where $\underline{n}$ is the normal vector and $Q_{\infty}$ is free stream velocity. Substituting equation (6) and equation (8) into equation (4), matrix equation (4) becomes a set of linear equations with unknown $\mu$. The doublet strength $\mu$ can therefore be evaluated by solving the matrix equation (4).

To represent the flow of blood across a valve implanted in the aorta we required to generate an internal flow analysis model. The internal flow modeling is accomplished by simulating the internal flow geometry as a closed tube with the panel normal vector pointing into the interior of the tube (Figure 2). The inlet cross section, the aortic wall, the outlet cross section and the valve leaflet are paneled by source/doublet elements. The wake flow downstream of the leaflet is simulated by doublet elements. Inflow and outflow to the tube (flow through a duct) may be prescribed as normal velocities on groups of panels. The inflow and outflow must be prescribed so that the continuity equation is satisfied, i.e.

$$
\int_{\delta A_{w}} \rho \underline{u}_{i n} \cdot d \underline{A}_{i n}=\int_{\delta A_{\text {out }}} \rho \underline{u}_{\text {out }} \cdot d \underline{A}_{\text {out }}
$$

where $\underline{u}_{\text {in }}$ and $\underline{u}_{\text {out }}$ are the velocities of inflow and outflow, $\underline{A}_{\text {in }}$ and $\underline{A}_{\text {out }}$ are the inlet and outlet cross section area and $\rho$ the blood density. Assuming uniform flow through the inlet and outlet cross section, inflow

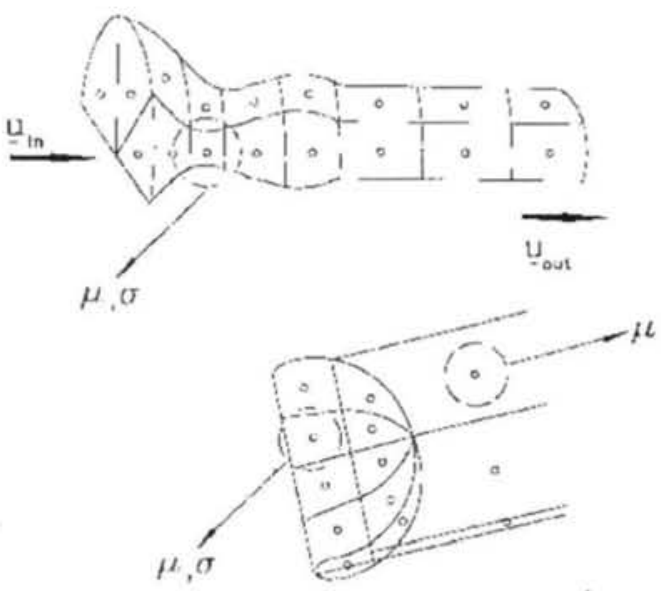

Fig. 2 Internal source/doublet analysis model of heart valve implanted in the aorta and outflow velocity therefore correspond to the relationship

$$
\underline{\boldsymbol{u}}_{\text {in }} \bullet \underline{\boldsymbol{A}}_{\text {in }}=\underline{\boldsymbol{u}}_{\text {out }} \bullet \underline{\boldsymbol{A}}_{\text {out }}
$$

The source values are determined by [12]

$$
\sigma=\frac{1}{4 \pi}\left(u_{\text {norm }}-\underline{n} \bullet \underline{u}_{\text {in }}\right)
$$

Here the normal velocity $u_{\text {norm }}$ at the panel control point is either zero (no flow through the surface, e.g. the aortic wall and the leaflet surface) or a defined value to simulate suction or blowing (e.g. $u_{\text {norm }}=\left|\underline{u}_{\text {in }}\right|$ on the inlet cross section and $u_{\text {norm }}=-\left|\underline{u}_{\text {out }}\right|$ on the outlet cross section) and the inlet velocity $\underline{u}_{\text {in }}$ is known. The wake doublet strength (equation 6) is related to the difference between that of upper and lower surface. Substituting equation (6) and equation (11) into equation (4), the matrix equation (4) with unknown doublet can now be written as

$\left|\begin{array}{cccc||c|c}C_{11} & C_{12} & C_{13} & \cdots \\
C_{21} & C_{22} & C_{23} & \cdots \\
C_{31} & C_{32} & C_{33} & \cdots \\
\cdots & \cdots & \cdots & \cdots\end{array}\right|$\begin{tabular}{|cccc}
$\mu_{1}$ \\
$\mu_{2}$ \\
$\mu_{3}$
\end{tabular}$|=| \begin{array}{cccc}B_{11} & B_{12} & B_{13} & \cdots \\
B_{21} & B_{22} & B_{23} & \cdots \\
B_{31} & B_{32} & B_{33} & \cdots \\
\cdots & \cdots & \cdots & \cdots\end{array}\left|\begin{array}{c}-\sigma_{1} \\
-\sigma_{2} \\
-\sigma_{3} \\
\cdots\end{array}\right|$

This linear dense matrix equation is solved by using the pre-conditioned $\mathrm{Bi}-\mathrm{CGSTAB}$ algorithm. Once the solution of equation (12) is obtained, velocities may be evaluated at positions within the fluid surrounding the solid surfaces. For example the velocity at the collocation point of panel $j, \underline{u}_{\mathrm{j}}$, is evaluated by

$$
\underline{u}_{j}=\nabla \phi_{j}+\underline{u}_{i n}
$$

Here $\underline{u}_{\text {in }}$ is the inlet flow velocity and $\phi_{j}$ is the perturbation potential at the collocation point of panel $j$ induced by all the singularity elements within the flow regime. The lift force $\left\lfloor\underline{\mid} \mid\right.$ and induced drag $\left|\underline{D}_{\text {id }}\right|$ acting on the leaflet surface can therefore be evaluated by

$$
\begin{aligned}
& |\underline{L}|=\sum_{j=1}^{N_{h}} \frac{1}{2} \rho\left|\underline{u}_{i n}\right|^{2} \cdot\left(1-\left|\underline{u}_{j}\right|^{2} /\left|\underline{u}_{i n}\right|^{2}\right) \cdot \Delta S_{j} \cdot \cos \beta_{j} \\
& \left|\underline{D}_{i d}\right|=\sum_{j=1}^{N_{h}} \frac{1}{2} \rho\left|\underline{u}_{i n}\right|^{2} \cdot\left(1-\left|\underline{u}_{j}\right|^{2} /\left|\underline{u}_{i n}\right|^{2}\right) \cdot \Delta S_{j} \cdot \cos \gamma_{j}
\end{aligned}
$$

where

$\Delta S_{j}$ is the area of the $j^{\text {th }}$ panel,

$\beta_{\mathrm{j}}$ is the angle between the panel normal vector and $\mathrm{z}$-axis,

$\gamma_{j}$ is the angle between the panel normal vector and $\mathrm{x}$-axis, and

$N_{\mathrm{b}} \quad$ is the number of source/doublet elements simulating the leaflet surface.

The associated lift force and induced drag coefficients 


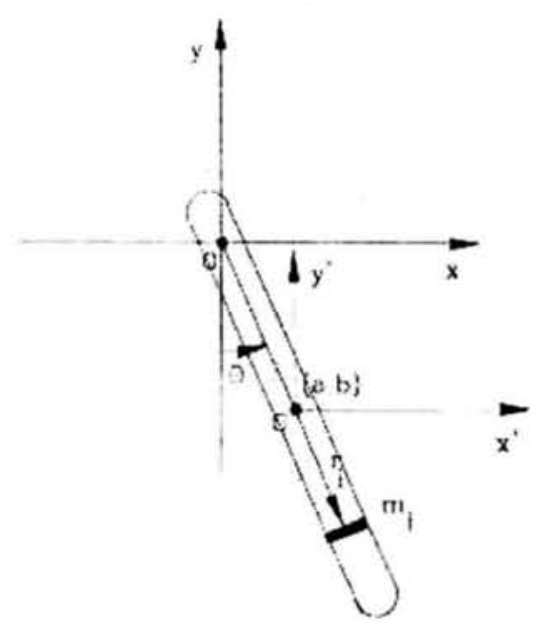

Fig. 3 A rigid body suspended and free to rotate about an axis

are evaluated by

$$
\begin{aligned}
& C_{L}=\frac{2|\underline{L}|}{\rho\left|\underline{u}_{i n}\right|^{2} A_{p}} \\
& C_{D_{\downarrow}}=\frac{2\left|\underline{D}_{i d}\right|}{\rho\left|\underline{u}_{i n}\right|^{2} A_{p}}
\end{aligned}
$$

Here $A_{p}$ is the leaflet projected area.

\subsection{Integrated Dynamic Model}

For the valve dynamic analysis, the leaflet is treated as rotational second order system [4]. The angular momentum balance is formulated to include the effects of the lift force, the drag force and the gravity force. In order to clarify the second order dynamic model, we present below the theory of rotating system.

Figure 3 shows a rigid body suspended and free to rotate about an axis. The axis is located at point $O$ and does not pass through the center of mass. The position of the body is specified by the angle $\theta$ between a vertical line and a line drawn from a point $O$ on the axis, through the center of mass $G$. In order to derive the system motion equation, we introduce the principle of angular momentum, that is the rate of change of the angular momentum of a system about a fixed axis is equal to the total moment of the external forces about the axis.

Let us first suppose that the rigid body consists of

$n$ particles. Let $\dot{\theta}$ be the instantaneous value of the angular velocity. Then the angular momentum of a particle of mass $m_{i}$ situated at a distance $r_{i}$ from the

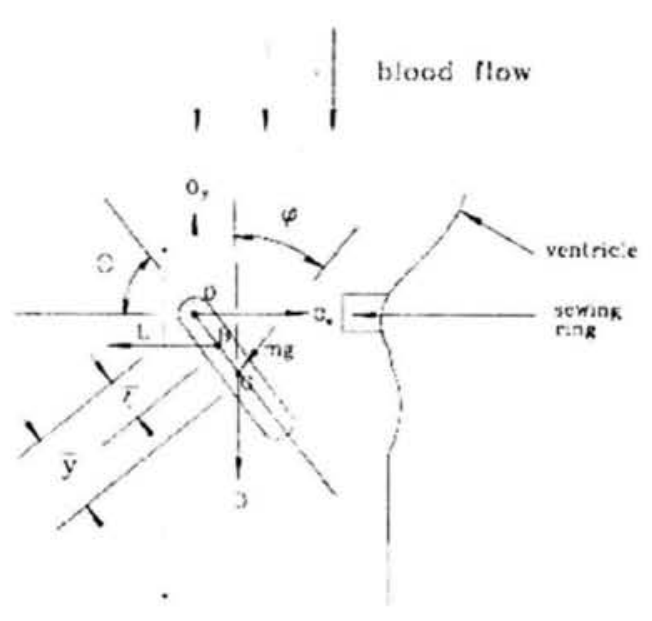

Fig 4. External forces acting on the valve leaflet

axis is $m_{i} r_{i}^{2} \dot{\theta}$, and so the angular momentum of the rigid body is

$$
\dot{\theta} \sum_{i=1}^{n} m_{i} r_{i}^{2}=I \dot{\theta}
$$

where $I=\sum_{i=1}^{n} m_{i} r_{i}^{2}$ is the moment of inertia.

In the case of a continuous distribution of matter, the definition of the moment of inertia passes over into

$$
I=\int r^{2} d m
$$

where the integration sign indicates the limit of a process in which the system is divided into a great number of very small parts and the sum taken; $d m$ is the mass of infinitesimal element, and $r$ is its distance from the line about which the momentom inertia is to be found.

Thus the equation of motion of the rotating system in Figure 3 can be expressed

$$
\sum_{i} M_{i}=\frac{d}{d t}(I \dot{\theta})=I \ddot{\theta}
$$

Here $\sum_{i} M_{i}$ is the summation of external moments about the axis. The evaluation of the moment of inertia can be simplified by using the theorem of parallel axis when the moment of inertia about a parallel line through the mass of center is known. Figure 4 shows the external forces acting on the valve leaflet. Here $O$ is the pivoting point, $G$ is the center of gravity and $B$ is the center of lift (a position about which the lift is assumed to act). The leaflet is simulated as a $2^{\text {nd }}$ order rotating system in a manner similar to Reif et al. [19], 
the equation of motion for the angular derivative of the valve leaflet is written as

$$
\ddot{I \theta}=\sum_{i} \underline{M}_{i}=\underline{M}_{L}+\underline{M}_{D}+\underline{M}_{C}
$$

$\mathrm{M}_{\mathrm{L}}=$ moment due to aerodynamic lift

$\underline{\mathrm{M}}_{\mathrm{D}}=$ moment due to drag

$\underline{\underline{M}}_{G}=$ moment due to gravity

Where $\theta$ is the opening angle of the leaflet and $I$ is the mass moment of inertia. The external moments are evaluated by:

$$
\begin{aligned}
& \underline{M}_{L}=\bar{\varsigma} L \sin \theta \\
& \underline{M}_{D}=\bar{y} D \cos \theta
\end{aligned}
$$

and

$$
\underline{M}_{B}=\bar{y} m g \cos (\theta-\phi)
$$

$\bar{\zeta}$ is the distance between the pivoting axis and the center of lift and $\bar{y}$ is the distance between the center of gravity and the pivoting axis. Here we assume the resultant lift acting on the center of lift and resultant drag acting on the center of gravity. The aerodynamic loads of lifting and induced drag are evaluated from the internal flow model rather than the data evaluated by Reif et al. [19]

The fluid model presented in the analysis of Reif ct al. [19] utilized classical flat plate aerodynamic theory and constrained the leaflet to operate in an infinite domain (external flow approach). This fluid flow model, although providing a simple and direct model, does not accurately simulate the influence from the aortic root, the stiffening ring and the other leaflet in the case of bi-leaflet valves. In order to provide an insight into the influence from the aortic root, the stiffening ring and the other leaflet in evaluating the dynamic characteristics, the internal flow model is therefore es-

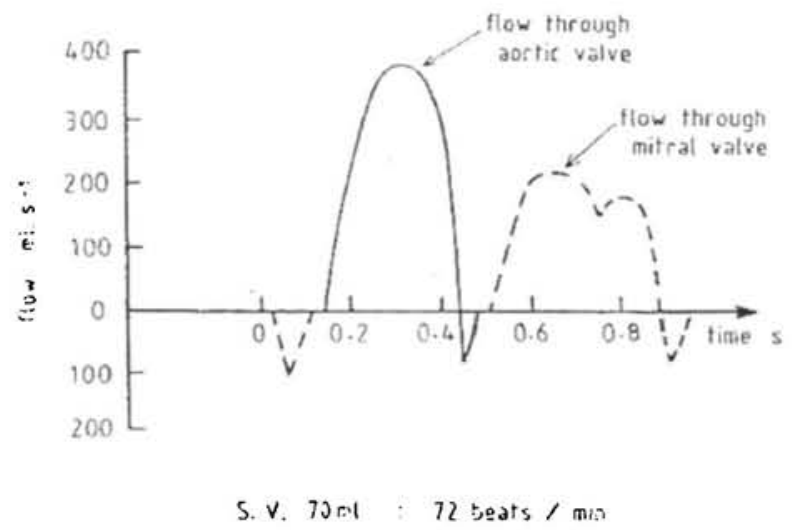

Fig. 5 Graph illustrating the variation of the flow rate through the heart [3] tablished to simulate the flow phenomenon.

For lifting bodies such as the leaflet at large angles of attack, flow separation takes place across the leaflet surface. The boundary layer separation can destroy the bound vortex on the body, thus destroying the lift which the leaflet generates. This is termed as 'stall condition' [19]. A stall condition is assumed to take place at $\theta=60^{\circ}$. Therefore, the mathematical form of lift and drag coefficients utilized in the integrated dynamic model is expressed as:

$$
\begin{aligned}
& C_{L}= \begin{cases}0 & \theta \leq 60^{\circ}\end{cases} \\
& C_{L}(\theta) \text { from the flow analysis } \quad \theta>60^{\circ} \\
& C_{D}= \begin{cases}C_{D S S} & \theta \leq 60^{\circ} \\
C_{L O}+C_{D,}(\theta) \text { from the flow analysis } & \theta>60^{\circ}\end{cases}
\end{aligned}
$$

Coefficients $C_{D S}$ and $C_{L O}$ are equal to 1.03 and 0.353 [19]. $C_{L}(\theta)$ and $C_{D i}(\theta)$ are evaluated by the steady internal flow model for an opening angle $\theta$. With fluid density $\rho$, entrance flow velocity $U$, the lift and drag forces are evaluated to be [19]:

$$
L=\frac{1}{2} \rho U^{2} A_{p} C_{L}
$$

and

$$
D=\frac{1}{2} \rho U^{2} A_{p} C_{D}
$$

Here $A_{p}$ is the projection area of the leaflet.

Figure 5 illustrates how the flow rate of blood through the heart varies as a function of time. Assuming that the changes in the cross sectional areas of the valves are negligible, the velocity of the blood will be a direct proportion of the flow rate at all times. Therefore the velocity profile will have a similar form to the flow rate. It can be seen that during the forward flow phase the aortic velocity function could, with a fair degree of accuracy, be approximated by a sine wave function of the form

$$
\begin{aligned}
& U=Q / A \\
& Q=\left\{\begin{array}{l}
0 \text { when leaflet is closed } \\
Q_{\max } \sin \left(\pi t / T_{s}\right) \quad \text { otherwise }
\end{array}\right.
\end{aligned}
$$

Where $A$ is the orifice area, $Q_{\max }$ is the instantaneous maximum flow rate, and $T_{s}$ is the systolic ejection period. The initial conditions are specified as 


$$
\begin{aligned}
& \theta(0)=\theta_{o} \\
& \frac{d \theta(0)}{d t}=0
\end{aligned}
$$

However the leaflet is restricted by the 'stop' mechanism at the maximum opening angle and by the stiffening ring at the minimum opening angle hence a complete model would be achieved by implementing an impact dynamic analysis. When the leaflet is opened to the maximum angle, it impacts against the 'stop' mechanism. The relation between angular velocities before and after impact is expressed as

$$
\omega_{2}=-e \omega_{1}
$$

where $e$ is the rebound coefficient, $\omega_{1}$ and $\omega_{2}$ are angular velocities before and after impact. We simply assume the value of rebound coefficient between the leaflet and the 'stop' mechanism at the fully open position to be 0.5 [4], i.e. $e\left(\theta=\theta_{\max }\right)=0.5$, to simulate the fluttering motion and zero between the leaflet and the stiffening ring, i.e. $e\left(\theta=\theta_{\min }\right)=0$, to avoid unexpected oscillation when the leaflet is closed.

In order to compare with other published results and to provide the correct physiological conditions, the cardiac output is assumed to be $3 \sim 10 \mathrm{l} / \mathrm{min}$ and the
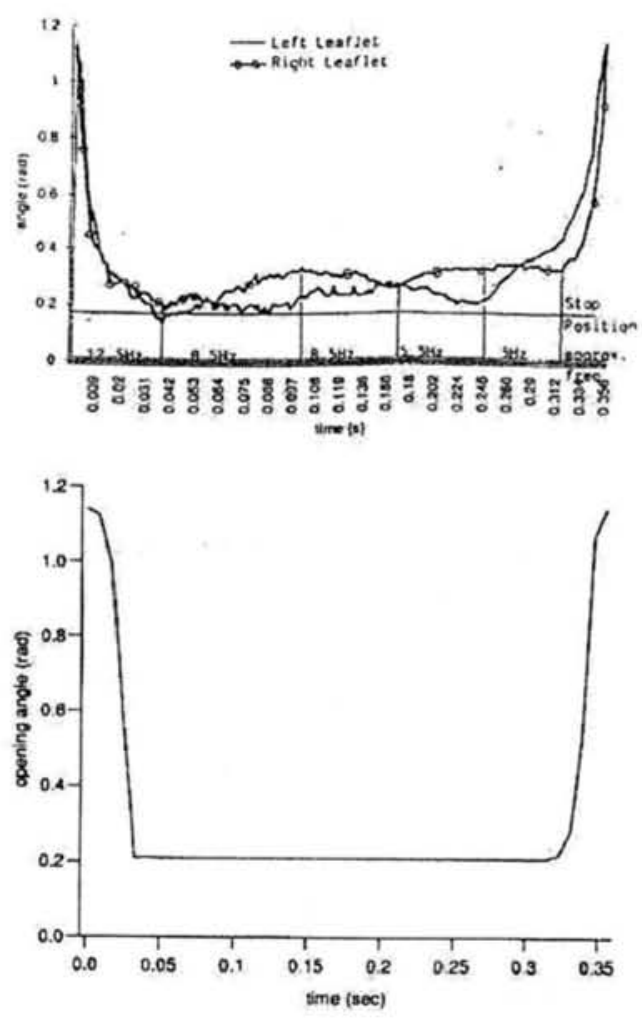

(b)

Fig.6 (a).Experimental opening characteristics of a $29 \mathrm{~mm}$ CarboMedics valve [3]; (b).A comparative study using the presented model flow across the valve is considered to resemble a sinewave mathematical form simulating the acceleration and deceleration phase of systole. The ratio of systolic time to the time for a complete cycle is assumed as 0.4 [4].

\section{RESULTS}

\subsection{Validation of the Internal Flow Model and the Dynamic Model}

To validate the presented dynamic model along with the internal flow model, a comparative study is investigated using a $29 \mathrm{~mm}$ CarboMedics valve. Figure 6(a) shows the results of a high speed video tape experimental study on the opening characteristics of a $29 \mathrm{~mm}$ CarboMedics valve when the cardiac output is $5 \mathrm{l} / \mathrm{min}$ (Canning [3]), here the vertical coordinate represents the angle subtended by the leaflet from the mainstream blood flow.

The leaflet opens to the maximum angle after about $39 \mathrm{msec}$ from the initiation of the flow pulse and a small amount of fluttering phenomenon is observed. A numerical simulation using the presented model for a $29 \mathrm{~mm}$ CarboMedics valve is shown in Figure 6(b) where the time required to open the leaflet is shown to be $42 \mathrm{msec}$ which is in excellent accord with the experimental result of Canning [3].

Figure 7(a) shows an in vitro experimental study on the dynamic behavior of a $21 \mathrm{~mm}$ St. Jude valve (van Steenhoven et al. [20]) when the cardiac output is approximately $3 \mathrm{l} / \mathrm{min}$.

The time required to open the leaflet is $65 \mathrm{msec}$ and $60 \mathrm{msec}$ to fully close the leaflet. The leaflet closes only for $5 \%$ of its cross-section area during systolic ejection. It therefore needs regurgitant flow to complete the closure. The ratio of the closing valve regurgitant flow to the main flow is approximately $2.5 \%$. A numerical simulation using the presented model for this case is shown in Figure 7(b). The time required to open the leaflet is $68 \mathrm{msec}$ and $60 \mathrm{msec}$ to fully close the leaflet when the orientation of gravity is perpendicular to the main flow. The ratio of the closing valve regurgitation flow to the main flow is approximately $2.6 \%$. These results are in excellent accordance with the experimental results of van Steenhoven et al. [20]

\subsection{A Simulation of the ST Jude Heart Valve}

The St Jude valve is a low-profile bi-leaflet prosthesis. In the fully open position the leaflets are designed to open to an angle of $85^{\circ}$. The leaflets are situated at upstream of the plane of the stiffening ring and this has a uniform square cross sectional area and a 'sharp' angular geometry in the inlet and outlet cross sections.

It has been pointed out in Yoganathan et al [24] 
that the flow through the St Jude valve is unobstructed and central and thus can achieve an 'optimal' effective valve area for a given available orifice area. A comparative study from the presented internal flow model and in vitro experimental data is illustrated below.

\section{(1).Separation point:}

As a fluid flows around the leading edge of the $\mathrm{St}$ Jude valve, a stagnation point is 'seen' [13] on the high pressure side of the leading edge of the leaflets. Flow separation occurs on the low pressure side of the leading edge of the leaflet since the fluid element at this location experiences a 'strong' adverse (unfavorable) pressure gradient which may 'weaken' the boundary layer sufficiently to cause separation.

Figure 8 shows the separation points behind the heart valve leaflet for various values of $\theta$. Decreasing $\theta$, the maximum opening angle, produces a leaflet orientation with a larger obstacle to the main blood flow and a corresponding recirculation zone behind the valve leaflet. The size of this recirculation vortex downstream of the valve gives an indication of the flow disturbance that would occur downstream of the valve [5]. In order to reduce the onset of the separation thus weakening the recirculatory eddies occurring downstream, an important trend of the valve design would be to allow the leaflet to have a curvature which
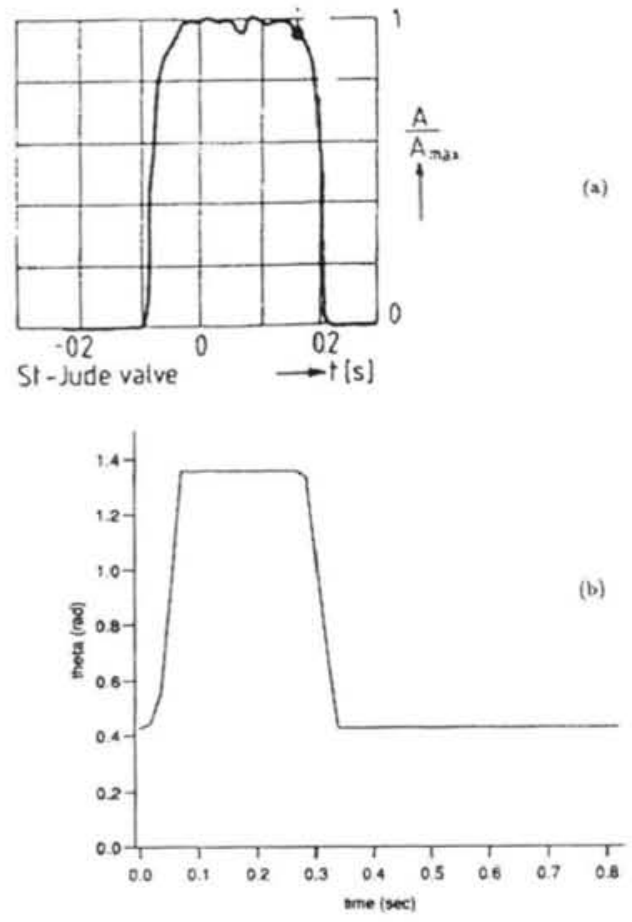

Fig 7. (a).Experimental opening characteristics of a $21 \mathrm{~mm}$ St Jude valve [20] ; (b).A comparative study using the presented model is parallel to the main flow streamline.

Consider the flow model from the stiffening ring surface of the St Jude valve, Figure 9 shows a flow visualization in the aortic root with the OS valve ring [14]. Flow separation begins at the leading edge of the ring due to the 'sharp' angular geometry. This leads to the formation of vortices behind the leading edge. The stream fills now only a small portion of the cross sectional area of the stiffening ring, and this in turn causes a higher pressure loss [5]. Additionally, this flow separation at the upstream corner will cause an additional unfavorable 'squeeze' effect on the out-flowing jet [5]. Meanwhile, the jets will be induced inboard by the vortical structures emanating from the trailing edge. The flow downstream of the ring is then strongly disturbed. The design of the ring geometry has, therefore, becomes rather crucial to provide a 'smooth' ring geometry which effectively places the separation point as far downstream as possible thus reducing the 'disturbing' effect on the orifice jet.

\section{(2). Shear stress}

Shear stress measurements obtained with in vitro studies for the flow downstream of the St Jude heart valve have been calculated by many investigators ([10],[22],[23],[24]), while there are very few studies on the measurement of leaflet surface shear stress due to difficulties in experimental techniques. Since the flow considcred was turbulent amongst all the experimental studies, the shear stress consists of a laminar and a turbulent component and is given by $\tau=\tau^{l}+\tau^{l}$. Here $\tau$ is total shear stress, $\tau^{l}$ is laminar shear stress and $\tau^{t}$ is turbulent shear stress. Typical values of shear stress downstream of the valves are listed in Table $I$. The fluid used in the in vitro studies of Yoganathan et al [24], Woo and Yoganathan [23] and Walker and Yoganathan [22] possess dynamic viscosity similar to blood $\left(\mu=3.5 \times 10^{-3} \mathrm{~kg} /(\mathrm{m} \bullet \mathrm{sec})\right)$. On the other hand, the fluid used in Hanle et al's [10] work had a dynamic viscosity of $\mu=10^{-3} \mathrm{~kg} /(\mathrm{m} \bullet \mathrm{sec})$, which is lower than the dynamic viscosity of whole blood. It should be noted that the shear stress measurements obtained with the in vitro studies of Table $I$ are for the flow downstream of the St Jude heart valve. None of the measurements provide the data of leaflet surface shear stress. Figure 10 shows the calculated leaflet shear stress for a $25 \mathrm{~mm}$ St Jude valve by the developed boundary layer model. Here the calculated laminar wall shear stress, $\tau_{w}$, is evaluated as a function of the non-dimensionalized streamwise coordinate along the center streamline, $x / R$, when the leaflet is at fully open position. The maximum laminar shear stress is approximately $103.02 \mathrm{~N} / \mathrm{m}^{2}$ when $\mu=3.5 \times 10^{-3} \mathrm{~kg} /(\mathrm{m} \bullet \mathrm{sec})$ and $29.43 \mathrm{~N} / \mathrm{m}^{2}$ when $\mu=10^{-3} \mathrm{~kg} /(\mathrm{m} \cdot \mathrm{sec})$ in the case of cardiac output equals $5 \mathrm{l} / \mathrm{min}$. 


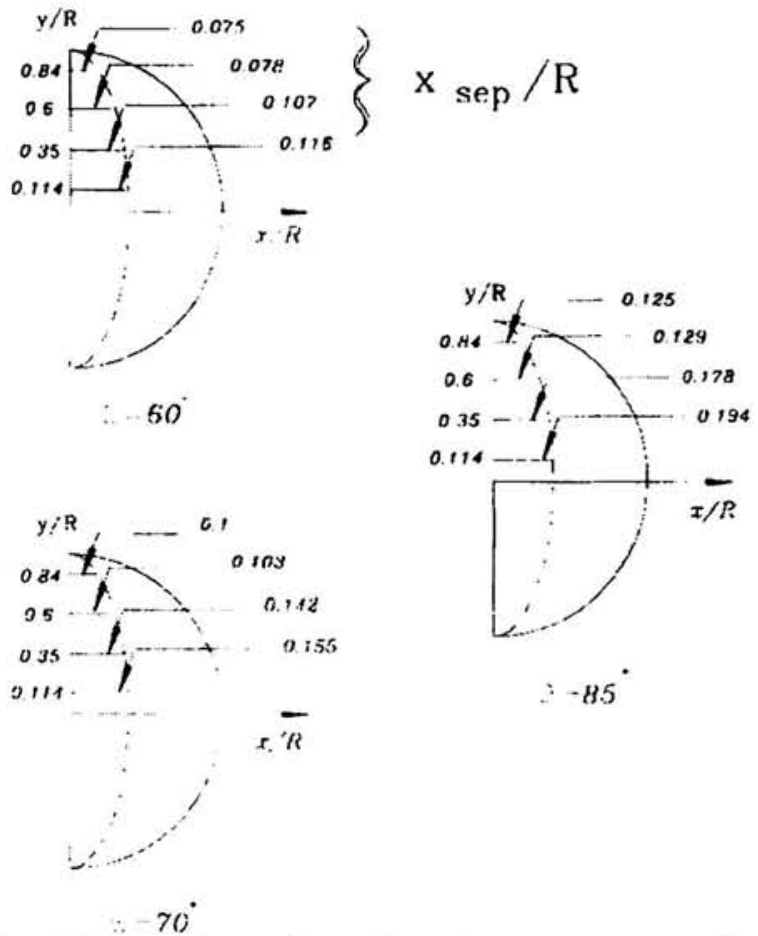

Fig. 8 Separation points along the center streamline of St Jude valve for various opening angles

\section{(3). Pressure loss}

The suggestion that valves be characterized in terms of energy loss, which is defined to evaluate the influence of the valve on heart work during each cardiac cycle, has attracted some attention for valve performance [17]. The energy loss $\left(\Delta E_{\text {energy,lass }}\right)$ would be defined as the product of forward flow rate $(Q)$ and pressure drop $(\Delta P)$ with the addition of energy loss due to leakage $\left(\Delta E_{\text {leakage }}\right)$, that is

$$
\Delta E_{\text {energy,loss }}=\int_{0} Q \cdot(\Delta P) d t+\Delta E_{\text {leakage }}
$$

where $T_{s}$ is the systolic ejection period. Clearly, the energy loss of the heart work is effectively corresponding to the pressure loss across the heart valve.

The pressure loss can be estimated by the presented boundary layer model. Table II illustrates pressure loss data estimated by the boundary layer model and in vitro measurements for St Jude valve. The associated pressure loss values listed on the far right of the table is evaluated by the boundary layer model. The pressure loss estimated from the boundary layer model is lower than that of in vitro experiment results. The underestimation of the pressure drop can be associated with a variety of reasons. For example, the pressure drop measurement in the work of Yoganathan et al [24] is made by the pressure difference of $19.5 \mathrm{~mm}$ upstream and $40.5 \mathrm{~mm}$ downstream the valve. This meas-
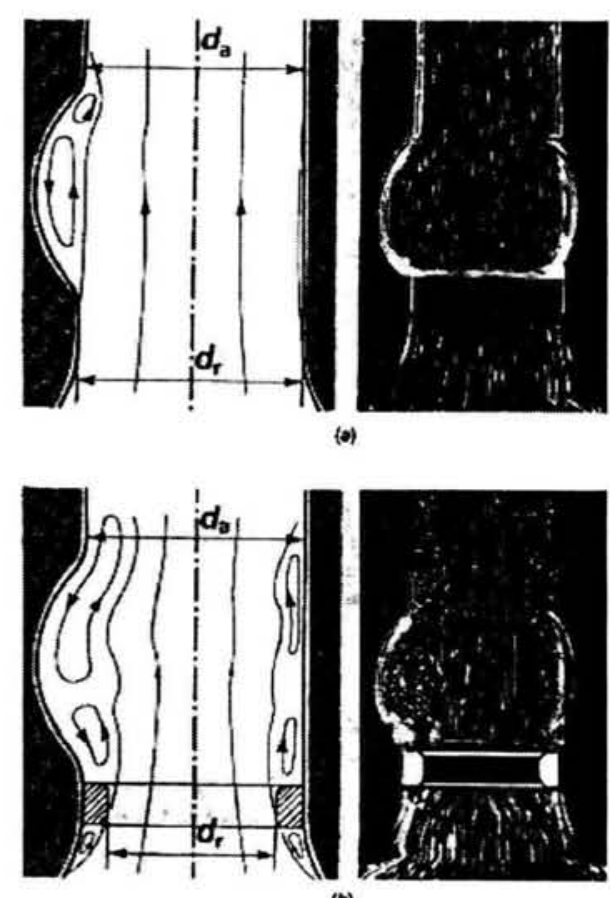

(b)

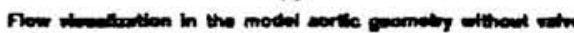

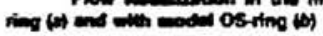

Fig. 9 A flow separation model from the stiffening ring of St Jude valve [14]

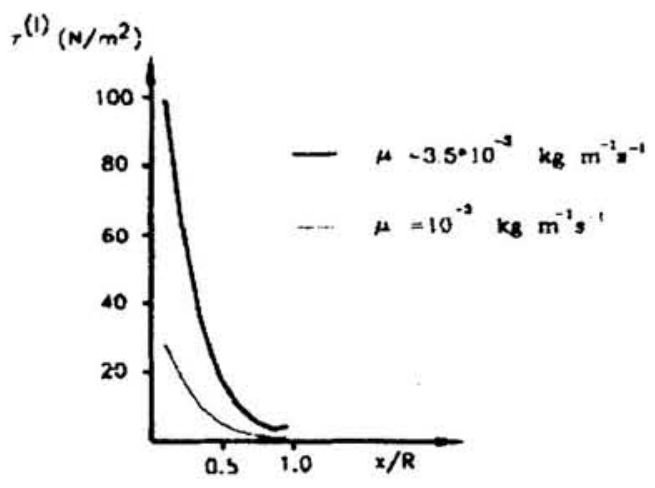

Fig. 10 Shear stress distribution across central chord of a $25 \mathrm{~mm}$ St Jude valve

urement results in a combined effect of pressure loss due to the heart valve and the aortic wall within the measurement. The other reasons which have been discussed by David and Hsu [5] are due to 'the additional pressure gradient required to overcome the timedependent acceleration of the fluid' and the 'simplicity' of the presented model in evaluating the effective ori- 

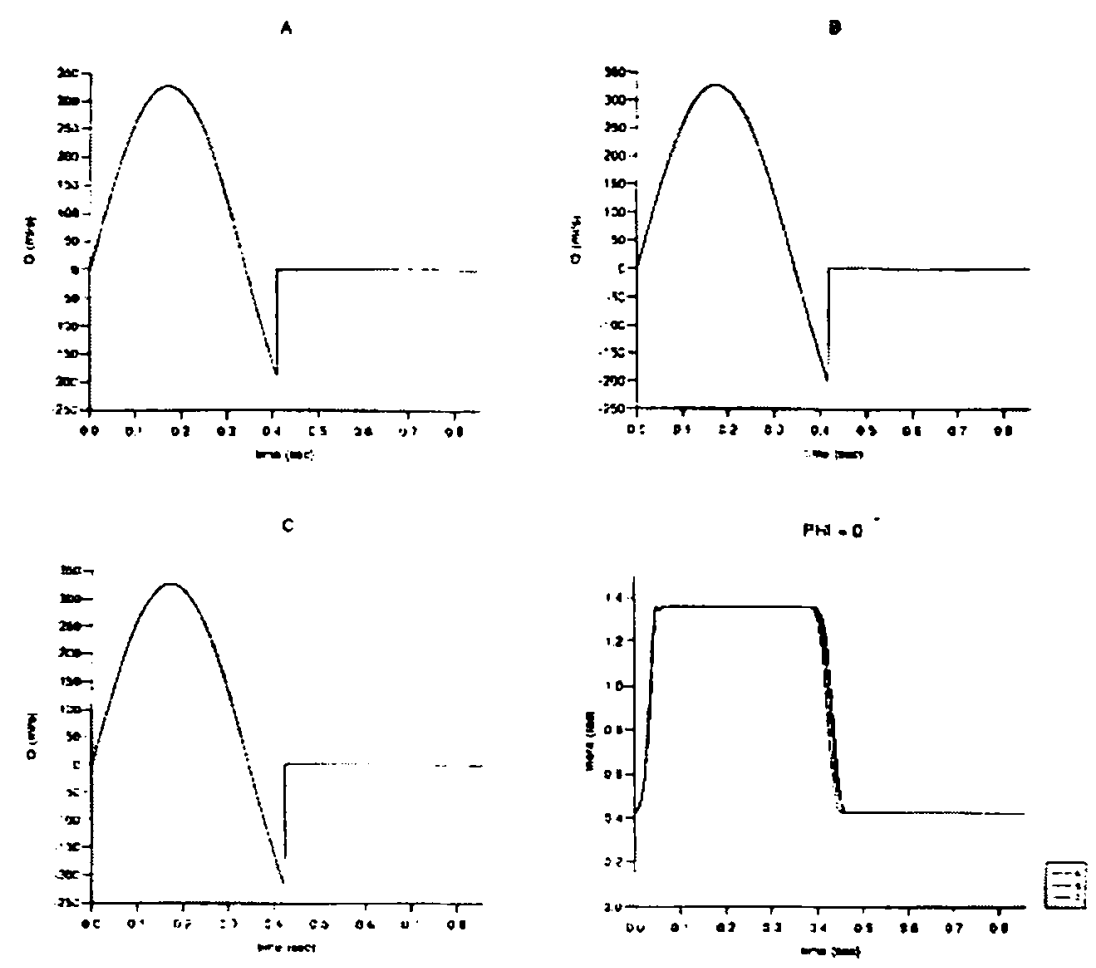

Fig. 11 Dynamic characteristics for $\phi=0^{\circ}$

fice area. However, we should note that these results are very encouraging for the 'simple' wake model since they predict important trends and can therefore be treated as part of the set of crucial design criteria.

\subsection{New Design Valve}

The bi-leaflet mechanical heart valve has been designed to produce a more uniform central flow pattern with reduced flow disturbance than tilting-disk valves. The disturbance is induced mainly by the geometric shape of the heart valve which includes the geometry of the leaflet and the stiffening ring. Since the heart valve geometry can influence the bulk blood flow, it is important that this aspect is modeled and analyzed. As has been suggested in David and Hsu [5], the leaflet geometry must be produced such that it provides a 'smooth' transition between the small valve diameter and larger aortic root diameter to reduce shear stress in the fluid and a leaflet shape with a sharp trailing edge that allows blood to flow downstream away from the leaflet in a smooth fashion. On the stiffening ring design, an internal flow analysis carried out by David and Hsu [5] has shown that the stiffening ring geometry affects the size of the recirculation zone behind the stiffening ring, the effective orifice area of the leaflet and the associated leaflet dynamic behavior. We present below an investigation for the effect of the location of the leaflets of a prototype heart valve design (we refer the reader to David and Hsu [5] for a full description of the prototype heart valve design). To investigate the effect of the location of the leaflet with respect to the stiffening ring in determining the corresponding dynamic characteristics, three cases of $29 \mathrm{~mm}$ new heart valve designs are studies. Case A corresponds to the leading edge of the leaflet located at the corner of the stiffening ring, case $\mathrm{C}$ the leading edge is located at the inlet cross section of the stiffening ring and case $B$ is with the leading edge located at the midpoint between that of case $A$ and $C$. The pivot axis is located at the center of the stiffening ring for all these three cases. In order to highlight the effect of the leaflet orientation with respect to the gravity vector on the valve dynamic characteristics, we compare two extreme cases: one for the case of the gravity vector is parallel to the main flow $\left(\phi=0^{\circ}\right)$; the other of the gravity vector opposite to the main flow $\left(\phi=180^{\circ}\right)$. Figure 11 shows the dynamic characteristics for A,B and $C$ with the gravity vector parallel to the main flow $\left(\phi=0^{\circ}\right)$.

The leaflet begins to close in the early phase of diastole. This is not an unexpected result due to the inertia of the mechanical prosthesis, additionally this has been observed experimentally (van Steenhoven et al [20]). However the required amount of the regurgitant flow to fully close the leaflet depend on the orientation of gravity and the location of the leaflet. 

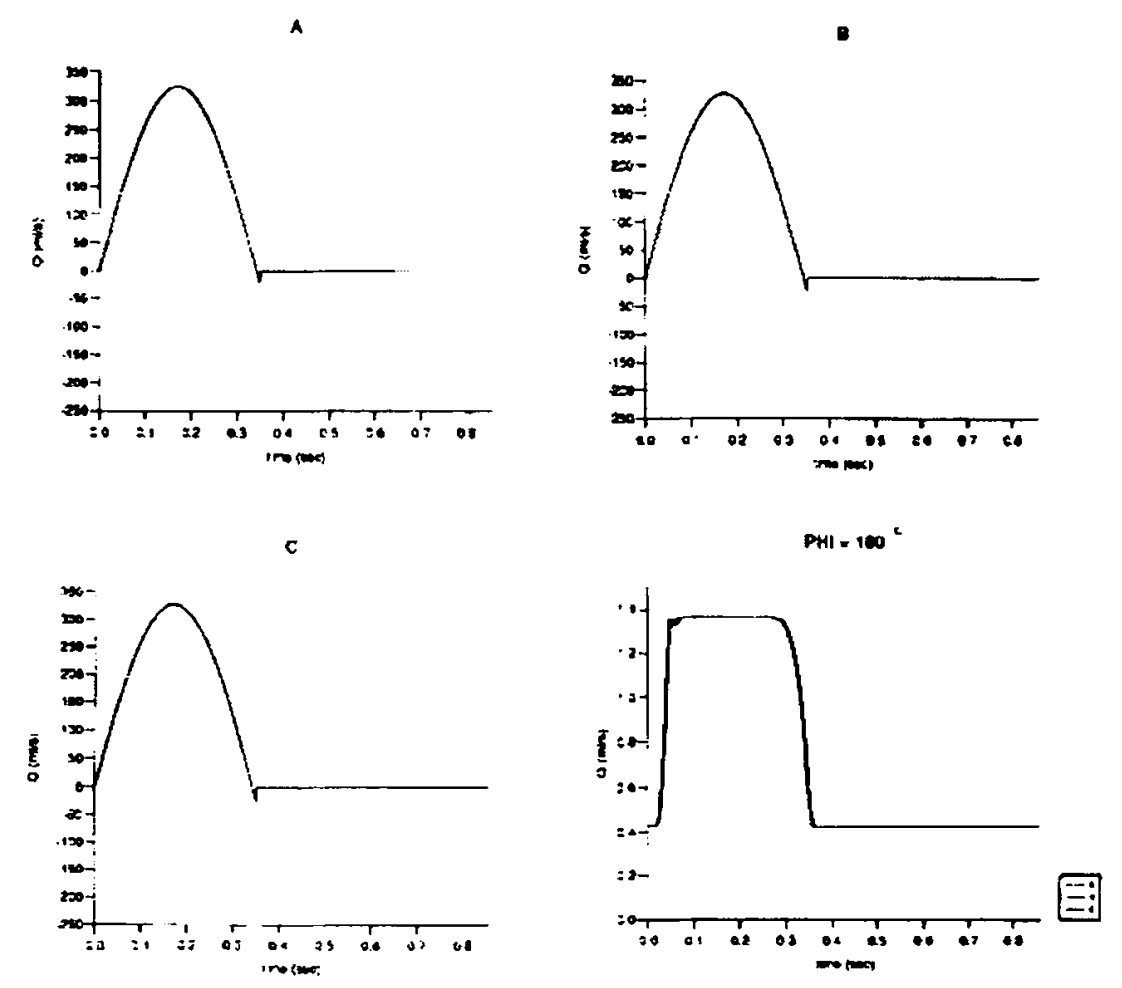

Fig. 12 Dynamic characteristics for $\phi=180^{\circ}$

Figure 11 shows a slightly different closing behavior for the cases $\mathrm{A}, \mathrm{B}$ and $\mathrm{C}$. The ratio of the closing valve regurgitation to the main flow is lower for case A $(9.12 \%)$ followed by case B $(10.48 \%)$ and highest for case $C(12.83 \%)$. There are two effects influencing the regurgitation, firstly due to the effective increase in the orifice arca at the position of the leaflet leading edge for case $\mathrm{C}$, therefore the lift is lower owing to the decreased strength of singularity elements maintaining the zero normal flow condition along the leaflet surface. Secondly, the magnitude of the radius vector from the pivot axis to the center of lift is smallest for case $C$. These two effects induce a lower lifting moment for case $\mathrm{C}$ to close the leaflet during the diastole thus increasing the closing valve regurgitation. Figure 12 shows the dynamic behavior for case $A, B$ and $C$ when gravity is opposite to the main for $\left(\phi=180^{\circ}\right)$. This is similar to the aortic valve opening when a person is in the standing position.

The ratio of the closing valve closing valve regurgitation to the main flow is significantly lower than that of $\phi=0^{\circ}$ (Figure 12). It is $0.103 \%$ for case $\mathrm{A}$, $0.134 \%$ for case $B$ and $0.17 \%$ for case $C$. This is not an unexpected result because the moment due to the gravity force tends to close the leaflet in this orientation thus making the valve close very gradually during the flow deceleration phase of systole mainly. After that only a minor regurgitant flow is necessary to complete the closure. The physiological criterion here is that a gradual valve closure with only a minor regurgitant flow decreases the occurrence of haemolysis and total energy loss, which takes into account the energy loss during forward and reverse flow, across the heart valve. On the consideration of minimizing the required regurgitant flow, the case A new design valve would be anticipated to provide a 'better' performance than that of case $B$ and case $C$.

\section{DISCUSSION AND CONCLUSION}

The internal flow model provides a correct evaluation of lift and induced drag and therefore the subsequent dynamic analysis. We concentrate the study of the valve design at the separation zone size behind the stiffening ring and the leaflets, the shear stress distribution over the leaflets, the location of the leaflets with respect to the stiffening ring and the corresponding dynamic behavior. It must be noted however that different criteria could lead to very different conclusions in determining the design parameters. The choice of the best overall valve depends on how much weight should be placed on each criterion.

On the ring geometry design we have pointed out that the 'squeeze' effect on the blood jets issuing through the three valve orifices of a bi-leaflet valve 
Table I. In vitro studies of shear stress downstream St Jude valve

\begin{tabular}{|c|c|c|c|c|}
\hline \multirow[b]{2}{*}{$\begin{array}{l}\text { St Jude } \\
\text { valve }\end{array}$} & \multicolumn{2}{|c|}{ Maximum total shear stress $\tau\left(N / m^{2}\right)$} & \multicolumn{2}{|c|}{$\begin{array}{l}\text { Maximum turbulent shear stress } \\
\tau^{(i)}\left(\mathrm{N} / \mathrm{m}^{2}\right)\end{array}$} \\
\hline & $\begin{array}{l}\text { Hanle et al }[10] \\
\text { (steady flow) }\end{array}$ & $\begin{array}{l}\text { Hanle et al [10] } \\
\text { Pulsatile flow }\end{array}$ & $\begin{array}{c}\text { Yoganathan et al } \\
{[25]} \\
\text { (Pulsatile flow) }\end{array}$ & $\begin{array}{l}\text { Woo et al [23] } \\
\text { (Pulsatile flow) }\end{array}$ \\
\hline $25 \mathrm{~mm}$ & 76 & 74 & 200 & 160 \\
\hline & & & $\begin{array}{c}\text { Yoganathan et al } \\
{[24]} \\
\text { (Steady flow) }\end{array}$ & $\begin{array}{l}\text { Walker et al [22] } \\
\text { (Pulsatile flow) }\end{array}$ \\
\hline $25 \mathrm{~mm}$ & & & 60 & 200 \\
\hline
\end{tabular}

Table II. Pressure loss across the St Jude valve

\begin{tabular}{|c|c|c|c|c|}
\hline St Jude valve & $\begin{array}{c}\text { Flow Rate } \\
\mathrm{Q}_{\text {ms }}(1 / \mathrm{min}) \\
\text { (Pulsatile flow) }\end{array}$ & $\begin{array}{c}\text { Fisher [8] } \\
\left(\mathrm{N} / \mathrm{m}^{2}\right)\end{array}$ & $\begin{array}{c}\text { Butterfield ct al [2] } \\
\left(\mathrm{N} / \mathrm{m}^{2}\right)\end{array}$ & $\begin{array}{l}\text { Wake model } \\
\left(\mathrm{N} / \mathrm{m}^{2}\right)\end{array}$ \\
\hline \multirow{3}{*}{$19 \mathrm{~mm}$} & 9 & 956 & -- & 571 \\
\hline & 12 & 1554 & -- & 1012 \\
\hline & 15 & 2471 & -- & 1581 \\
\hline \multirow{3}{*}{$21 \mathrm{~mm}$} & 9 & 380 & -- & 308 \\
\hline & 12 & 683 & -- & 547 \\
\hline & 15 & 1063 & -- & 855 \\
\hline \multirow{3}{*}{$23 \mathrm{~mm}$} & 12 & & 431 & 352 \\
\hline & 18 & & 1162 & 797 \\
\hline & 21 & & 1461 & 1089 \\
\hline St Jude valve & $\begin{array}{c}\text { Flow velocity } \\
\text { U (m/s) } \\
\text { (Steady flow) }\end{array}$ & $\begin{array}{c}\text { Yoganathan et al } \\
{[24]\left(\mathrm{N} / \mathrm{m}^{2}\right)}\end{array}$ & & $\begin{array}{l}\text { Wake model } \\
\left(\mathrm{N} / \mathrm{m}^{2}\right)\end{array}$ \\
\hline \multirow{3}{*}{$27 \mathrm{~mm}$} & 0.2 & 180 & & 131 \\
\hline & 0.3 & 387 & & 290 \\
\hline & 0.4 & 645 & & 461 \\
\hline \multirow{3}{*}{$25 \mathrm{~mm}$} & 0.2 & 225 & & 167 \\
\hline & 0.3 & 450 & & 342 \\
\hline & 0.4 & 750 & & 562 \\
\hline
\end{tabular}

should be minimized. This can be achieved by either having no separation flow around the ring surface or that separation should occur as far downstream as possible. Additionally it should be noted that on the manufacture viewpoint the ring geometry would be designed with a 'minimum' curvature of the ring surface to avoid a 'sharp' angular surface shape. This geometry can be achieved by either a 'thoughtful' use of the modeling geometry for ring geometry or an increase on the width of the ring. The increased ring width would provide geometry 'buffer' in order to achieve a 'smooth' transition geometry between the ventricle and the aorta.

In the in vitro closing behavior analysis for BjorkShiley, St Jude and Hancock heart valve prostheses carried out by van Steenhoven et al [20], it has been shown that the mechanical prostheses mainly close due to the regurgitant flow in the early phase of diastole while the natural valve closure is gradual and has al- ready started during the deceleration phase of systolic ejection, consequently only a minor regurgitant flow in the valve is required to complete valve closure. Due to the higher regurgitant volumes of the mechanical prostheses, the shear stress and the occurrence of hemolysis are correspondingly higher. However, it must be noted that the regurgitant volumes depends very much on the oricntation of the gravity force. The analysis of the presented new heart valve design leads to a conclusion that the difference of the ratio of the valve closing regurgitation to the main flow between the case of $\phi=0^{\circ}$ and $\phi=180^{\circ}$ can be as high as $12 \%$. This is because the moment due to gravity force tends to close the leaflet in the orientation of $\phi=180^{\circ}$ in contrast to the case of $\phi=0^{\circ}$ thus making the valve close very gradually during the flow deceleration phase of systole therefore only a minor regurgitant flow is required to complete the closure. However, it should be noted that a favorable (or unfavorable) effect 
of the gravity vector on the leaflet closing characteristics would induce an opposite influence on the valve opening characteristics. Hence we mention that the leaflet should be manufactured with a rather light material to avoid the unstable influence on the heart work due to the human's physical actions.

\section{ACKNOWLEDGEMENT}

We acknowledge the support of National Science Council under the grand NSC 90-2213-E-218-006 in developing this study.

\section{REFERENCES}

1. Black MM, Drury PJ and Tindale WB: Twenty-five years of heart valve substitutes: a review. J. Royal Society Med. 1983; 76: 667-681.

2. Butterfield M, Fisher J, Davies GA and Spyt TJ: Comparative study of the hydrodynamic function of the CarboMedics valve. Ann. Thorac. Surg. 1991; 52: 815-820.

3. Canning P: The prediction of the dynamic performance of artificial heart valves. Leeds University Mechanical Engineering Department, final year project, 1994.

4. Cheon GJ and Chandran KB: Dynamic behavior analysis of mechanical mono-leaflet heart valve prostheses in the opening phase. ASME Trans: J. Biomech. Eng. 1993; 115: 279-283.

5. David $\mathrm{T}$ and $\mathrm{Hsu} \mathrm{CH}$ : Interactive design of mechanical bi-leaflet heart valve. $2^{\text {nd }}$ World Congress Biomechanics, Amsterdam, 1994.

7. Dubini G, Pietrabissa $R$ and Fumero R: Computational fluid dynamics of artificial heart valves. Int. J. Artif. Organs 1991; 14: 338-342.

8. Fisher J: A comparative study of the hydrodynamic function of the size $19 \mathrm{~mm}$ and $21 \mathrm{~mm} \mathrm{St} \mathrm{Jude}$ medical haemodynamic plus bi-leaflet heart valves. Joumal of Heart Valve Disease 1994; 3(1): 75-80.

9. Greenfield $\mathrm{H}$ and Kolff W: The prosthetic heart valve and computer graphics. JAMA 1972; 219: 69-74.

10. Hanle DD, Harrison EC, Yoganathan AP, Allen DT and Corcoran WH: In vitro flow dynamics of our prosthetic aortic valves: a comparative analysis. J. Biomechanics 1989; 22: 597-607.

11. Ito $\mathrm{H}$ and Nanbu $\mathrm{K}$ : Flow in rotating straight pipes of circular cross section. ASME Trans.: J. Basic Eng. 1971; 93: 383-394.

12. Katz $\mathbf{J}$ and Plotkin A: Low speed aerodynamics, from wing theory to panel methods. Eds., McGrawHill, New York, 1991.
13. King M: Computational and experimental studies of flow through a bi-leaflet mechanical heart valve. Leeds University $\mathrm{PhD}$ Thesis, Leeds University UK, 1994.

14. Knoch M, Reul K, Kroger R and Rau G: Model studies at mechanical aortic heart valve prosthesesPart I: Steady-state flow field and pressure loss coefficients. ASME Trans.: J. Biomechanical Eng. 1988, 110: 334-343.

15. McQueen DM and Peskin CS: Computer-assisted design of butterfly bi-leaflet valves for the mitral position. Scand. J. Thor. Cardiovasc. Surg. 1985; 19: 139-148.

16. McQueen DM and Peskin CS: A three dimensional computational method for blood flow in the heart, part II contractile fibers. J. Computational Physics 1989; 82: 289-297.

17. Nolan SP: Defining valve performance: importance and difficulties in testing heart valve replacement devices. J. Medical Engineering \& Technology 1992; 16(1): 1-3.

18. Prabhu AA and Hwang NHC: Dynamic analysis of flutter in disc type mechanical heart valve prostheses. J. of Biomechanics 1988; 21(7): 585-590.

19. Reif TH, Schutle TJ and Hwang NHC: Estimation of the rotational undamped natural frequency of bileaflet cardiac valve prostheses. ASME Trans: J. Biomech. Eng. 1993; 115: 389-395.

20. van Steenhoven AA, van Duppen JAG, Cauwenberg JWG and van Renterghem RJ: In vitro closing behavior of Bjork-Shiley, St Jude and Hancock heart valve prostheses in relation to the in vivo recorded aortic closure. J. Biomechanics 1982; 15(11): 841-848.

21. Tansley GD, Edwards RJ and Gentle CR: Role of computational fluid mechanics in the analysis of prosthetic heart valve flow. Medical and Biological Eng. And Computing. 1988; 20: 175-185.

22. Walker PG and Yoganathan AP: In vitro pulsatile flow hemodynamics of five mechanical aortic heart valve prostheses. Eur. J. Cardio-thorac. Surg. 1992; 6: s113-s123.

23. Woo YR and Yoganathan AP: In vitro pulsatile flow velocity and turbulent shear stress measurements in the vicinity of mechanical aortic heart valve prostheses. Life Support System 1985; 3: 283-312.

24. Yoganathan AP, Chaux A, Gray RJ, deRobertis M and Matloff JM: Flow characteristics of the St Jude prosthetic valve: An in vitro and in vivo study. Artificial Organs 1982; 6(3); 288-294.

25. Yoganathan AP, Woo YR and Hsing WS: Turbulent shear stress measurements in the vicinity of aortic valve prostheses. J. Biomechanics 1986; 19:433-442. 